rings of carbon atoms. These compounds have the formula $\mathrm{C}_{7} \mathrm{H}_{14}$ and, in nearly all petroleum samples examined, their abundances relative to those of the $\mathrm{C}_{7} \mathrm{H}_{16}$ compounds are consistent with the prediction of Mango's kinetic scheme. On mechanistic grounds, it is expected that the alkane ring systems are more stable than the linear structures, and this is confirmed by results of Mango's differential cracking experiments ${ }^{1,6}$.

If the subsurface lifetimes of hydrocarbons were as brief as suggested by earlier estimates, we would expect to find many oils in which relative abundances of cyclic structures had increased as the less stable straightchain compounds were degraded. On the contrary, in the present report $^{1}$, Mango shows that, even though the expected enrichment of cyclic structures can be generated in

\title{
Misaligned viewpoints
}

\section{A. J. Parker}

THE use of binocular vision to recover threedimensional structure depends upon the geometrical fact that each eye acquires a slightly different view of the world. Thus, differences in depth within the scene are transformed into small differences between the images on each retina, notably in the relative horizontal positions of features $^{1}$. On page 156 of this issue $^{2}$, DeAngelis, Ohzawa and Freeman offer evidence that the fine structure of receptive fields in the visual cortex is shaped to exploit this information.

For the brain to exploit horizontal disparities, the correspondence between features on each separate retina must somehow be established. Physiologically, one way to do this is to have binocular detectors, which are tuned to identi-

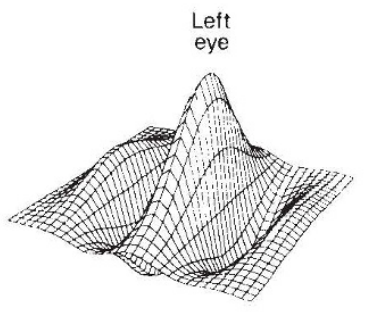

to only a limited range of spatial frequencies within the image ${ }^{5}$.

These findings are captured by a model in which the receptive field is described by a two-dimensional sinusoidal wave packet cal properties of the image but are selectively sensitive to small geometric differences in horizontal position. Such detectors have been found ${ }^{3}$, but the present evidence indicates that the mechanisms for establishing similarity and binocular correspondence may be more diverse than has been supposed.

The new analysis provides more detail about the spatial structure of the visual receptive fields of neurons in cortical area V1 (striate cortex) of cats. This is the first stage in the visual pathway where binocular interactions are readily observed and many of the neurons can be excited to some degree by stimulation through either eye ${ }^{4}$. Classically, these receptive fields are known to be restricted in spatial extent, selective for the orientation of image features ${ }^{4}$ and sensitive gaussian, known as a Gabor function - see figure $)^{6,7}$. The detailed shape of the receptive field is determined by several parameters in the model, but the new evidence suggests that the phase of the sinusoid may be crucial for the disparity selectivity of a particular population of cortical cells. For some binocular cortical cells, the inference is that, when receptive fields are measured through the left and right eyes, the measurements are identical except in this parameter.

A shift in the phase, leaving other parameters constant, changes the locations of the peaks and troughs of the receptive fields in the left and right eyes (see figure). This would give the cell some sensitivity to binocular disparity and, indeed, it is possible to the laboratory by extreme thermal stress, it is almost never observed in nature. The structural evidence from field observations - that enrichment of cycloalkanes is nearly absent - thus comes down strongly on the side of subsurface lifetimes. tories, Department of Geological Sciences, Indiana University, Bloomington, Indiana 47405, USA.

1. Mango, F. D. Nature 352, 146-148 (1991).

2. Mango, F. D. Science 273, 514-517 (1987)

3. Mango, F. D. Geochim. cosmochim. Acta 54, 1315-1323 (1990).

4. Quigley, T. M. \& Mackenzie, A. S. Nature 333, 549-552 (1988).

5. Barker, C. Am. Assoc. Petrol. Geol. Bull. 74, 1254-1261 (1990).

6. Mango, F. D. Geochim. cosmochim. Acta 54, 23-27 (1990). the new laboratory evidence for long

J. M. Hayes is in the Biogeochemical Labora-

express binocular disparities as shifts in the spatial phase of a sinusoidal signal, in much the same way that interaural time differences in the auditory system can be expressed as a temporal phase lag.

The scheme proposed by DeAngelis et al. ${ }^{2}$ bears upon the general problem of phase recovery in signal processing. Indeed, there are specific computational proposals for treating stereoscopic disparities as if they were spatial phase differences ${ }^{8}$. Similarly, there are proposals for the use of quadrature pairs of filters in the $x-t$ spatiotemporal domain for the detection of visual motion ${ }^{9,10}$.

Now that the new conceptual scheme has been put forward, it is important to understand its relationship with earlier results $^{3}$, including those of Freeman's group $^{11}$. That work used truly binocular visual stimulation and it would be good to see the reverse correlation technique ${ }^{2,11}$ applied binocularly. The present measures have provided no information on the possibility of interocular differences in the location of the peak of the gaussian envelope (see Fig. $1 a$ of the paper on page 157). Yet the phase differences observed in this work cannot be translated into stereoscopic disparity without knowing the location of the envelope peak as well.

It would be ironic if the position of the envelope peak were found to covary with the phase difference, but such an organization could actually be produced by a developmental mechanism that attempts to construct binocular receptive fields whose left and right eye inputs are as closely matched as possible, thus presumably causing the cell to be tuned to zero disparity. If the initial distribution of the influences of the left and right eyes were misaligned, then phase differences could be induced by a developmental mechanism that refines binocular connections based on the degree of binocularly correlated (visually driven) activity. Such an outcome would return the cell towards being tuned to zero disparity, and indeed it is a puzzle to understand what kind of developmental process could lead to the appearance of controlled degrees of dissimilarity between receptive field structures for the left and right eyes.

(sinusoid multiplied by a two-dimensional
A. J. Parker is in the University Laboratory of Physiology, University of Oxford, Parks Road, Oxford OX1 3PT, UK.

1. Wheatstone, C. Phil. Trans. R. Soc. 128, 371-394 (1838)

2. DeAngelis, G.C., Onzawa, I. \& Freeman, R.D. Nature 352, 156-159 (1991).

3. Barlow, H.B., Blakemore, C.B. \& Pettigrew, J.D. J. Physiol. 193, 327-342 (1967).

4. Hubel, D.H. \& Wiesel, T.N. J. Physiol. 160, 106-154 (1962).

5. Cooper, G.F. \& Robson, J. IEE Conf. Pub. 42 (IEE/NPL Conf. on Pattern Recognition, 1968).

6. Daugman, J. Vision Res. 20, 847-856 (1980).

7. Marčelja, S. J. Opt. Soc. Am. 70, 1297-1300 (1980).

8. Jenkin, M.R. Tech. Rep. RBCV-TR-88-22 (Dept Computer Sci., Univ. Toronto, 1988).

9. Watson, A.B. \& Ahumada, A. J. Opt. Soc. Am. 2, 322-342 (1985).

10. Adelson, E.H. \& Bergen, J.R. J. Opt. Soc. Am. 2 284-299 (1985).

11. Freeman, R.D. \& Ohzawa, I. Vision Res. 30, 1661-1676 (1990) 